\title{
Local Non-injectivity for Weighted Radon Transforms
}

\author{
Jan Boman
}

\begin{abstract}
A weighted plane Radon transform $R_{\rho}$ is considered, where $\rho(x, L)$ is a smooth positive function. It is proved that the set of weight functions $\rho$, for which the map $f \mapsto R_{\rho} f$ is not locally injective, is dense in the space of smooth positive weight functions.
\end{abstract}

\section{Introduction}

We shall consider a weighted plane Radon transform

$$
R_{\rho} f(L)=\int_{L} f(x) \rho(x, L) d s,
$$

where $L$ denotes an arbitrary line in the plane, $d s$ is arc length measure on $L$, and $\rho(x, L)$ is a given, smooth, positive function defined on the set of pairs $(x, L)$ where $x=\left(x_{1}, x_{2}\right)$ is a point on $L$. It is well known that $R_{\rho}$ is not always injective on the set of functions $f$ with compact support [Bo1]. On the other hand, if $\rho(x, L)$ is positive and real analytic, it is known that $R_{\rho}$ is not only injective on compactly supported functions but also locally injective in the following sense. Assume that the function $f$ (continuous, say) is supported in the set $\left\{\left(x_{1}, x_{2}\right) ; x_{2} \geq \delta x_{1}^{2}\right\}$ for some $\delta>0$ and that $R_{\rho} f(L)=0$ for all lines $L$ in a neighborhood of the line $x_{2}=0$; then $f=0$ in some neighborhood of the origin $[\mathbf{B Q}]$. Hence the set of $\rho$ for which $R_{\rho}$ is locally injective is dense in the set of smooth, positive weight functions. Here we shall show that the set of $\rho$ for which $R_{\rho}$ is not locally injective is also dense (Theorem 1.3). We shall do this by presenting a simplified version of the construction in [Bo1] and extending it to a dense set of $\rho$. By contrast, it is well known that the set of positive $\rho$ for which $R_{\rho}$ is globally injective is open in the $C^{1}$ topology. Indeed, the inverse of $R_{\rho}$, if it exists, must be bounded in certain Sobolev norms, and it is a simple fact that the set of operators with bounded inverse must be open. It follows that the set of $\rho$ for which $R_{\rho}$ is globally injective is open and dense in the set of positive weight functions.

The interest in the mathematical theory of weighted Radon transforms began with the invention of the Single Photon Emission Computed Tomography (SPECT)

1991 Mathematics Subject Classification. Primary 44A12.

Key words and phrases. weighted Radon transform, local injectivity.

(c)0000 (copyright holder) 
in the 1970s. As is well known, SPECT requires the inversion of the so-called attenuated Radon transform, which corresponds to the case when

$$
\rho(x, L)=\exp \left(-\int_{L(x)} \mu(z) d s_{z}\right),
$$

where $L$ is an oriented line, $L(x)$ denotes one of the components of $L \backslash\{x\}$, and $\mu(x)$ is a known function (attenuation coefficient) defined on the plane. A breakthrough was achieved by Alexander Bukhgeim et al. $[\mathbf{A B K}]$ and independently by Roman Novikov [No] with proofs of inversion formulas for the attenuated Radon transform $R_{\rho}$, where $\rho$ has the form (1.2). Since then many articles on the subject have appeared, for instance $[\mathbf{N a}],[\mathbf{B S}],[\mathbf{B a 1}],[\mathbf{F i}],[\mathbf{K B}],[\mathbf{B a} 2],[\mathbf{F o}]$.

Gindikin recently obtained an inversion formula for a somewhat related, but different class of smooth $\rho,[\mathbf{G}]$. For the class of $\rho$ considered in $[\mathbf{G}]$, local injectivity was proved in $[\mathbf{B o 2}]$. For constant $\rho$ local injectivity was first proved by Strichartz $[\mathbf{S}]$

It seems not to be known if local injectivity holds for the class of attenuated Radon transforms.

In our study of local injectivity for the plane weighted Radon transform we shall denote the coordinates in $\mathbf{R}^{2}$ by $(x, y)$ and we shall parametrize the lines as follows: $L(\xi, \eta)$ will denote the line $y=\xi x+\eta$ in the $x y$ plane, and the weight function will be written $\rho(x, \xi, \eta)=\rho(x, L(\xi, \eta))$. Thus $R_{\rho}$ will be defined by

$$
R_{\rho} f(\xi, \eta)=\int_{\mathbf{R}} f(x, \xi x+\eta) \rho(x, \xi, \eta) d x .
$$

THEOREM 1.1. There exists a smooth, positive function $\rho(x, \xi, \eta)$ defined on $\mathbf{R}^{3}$ and a smooth function $f(x, y)$, supported in $|x| \leq y$ with $(0,0) \in \operatorname{supp} f$ such that

$$
R_{\rho} f(\xi, \eta)=0
$$

for all $|\xi|<1, \eta<1$.

Using Theorem 1.1 we can rather easily construct a counterexample to global injectivity.

Corollary 1.2. ([Bo1, Theorem]) There exists a smooth function $f$, supported in the unit disk, not identically zero, and a smooth positive weight function $\rho(x, y, L)$ such that $R_{\rho} f(L)=0$ for all lines $L$ in the plane.

Proof of Corollary 1.2. Note that the weight function cannot be written $\rho(x, L)$ for lines $L$ that are parallel to the $y$-axis. Let $D$ be the open unit disk and let $K$ be the compact subset of $D$ consisting of the starshaped region in Figure 1 with the six shaded triangular areas omitted. Denote the bottom shaded triangle by $T$. Using Theorem1.1 we can define a smooth function $f$ in $T$ and a smooth, positive function $\rho(x, y, L)$ such that the bottom point of $T$ is in supp $f$ and $R_{\rho} f(L)=0$ for all lines $L$ intersecting $T$ but not intersecting $K$. Rotating the coordinate system we can do the same for the other shaded areas. Thus we have defined

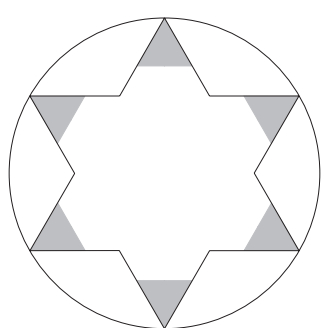

Figure 1 $f(x, y)$ and $\rho(x, y, L)$ for $(x, y) \notin K$ and lines $L$ not intersecting $K$ such that the support of $f$ meets the boundary of $D$ and $R_{\rho} f(L)=0$ for all lines not intersecting 
$K$. The rest of the proof follows a simple idea in [Bo1]. Extend $f$ to the entire disk $D$ so that $f$ takes positive as well as negative values on every line intersecting the interior of $D$. Then for each line $L$ intersecting $K$ it is easy to extend the function $(x, y) \mapsto \rho(x, y, L)$ as a positive function so that the integral of $f(x, y) \rho(x, y, L)$ over $L$ becomes zero. It is also easy to do this in such a way that the function $(x, y, L) \mapsto \rho(x, y, L)$ is smooth. See Lemma 2 in [Bo1] for details. This completes the proof.

Let $\mathcal{W}$ be the set of smooth weight functions $\rho(x, \xi, \eta)$ defined on the set $[-1,1]^{3} \subset \mathbf{R}^{3}$. On $\mathcal{W}$ we consider the usual $C^{\infty}$ topology, that is, the topology defined by the family of seminorms $p_{m}$, where

$$
p_{m}(\rho)=\sup \left\{\left|\partial_{\xi}^{r} \partial_{\eta}^{s} \partial_{x}^{t} \rho\right| ; r+s+t \leq m\right\}, \quad m=0,1, \ldots
$$

Here is the main result of this paper.

THEOREM 1.3. Let $\rho_{0}(x, \xi, \eta)$ be an arbitrary smooth, positive function in $\mathcal{W}$. For an arbitrary neighborhood $V$ of $\rho_{0}$ there exists a weight function $\rho \in V$ and a function $f$ supported in $|x| \leq y$ with $(0,0) \in \operatorname{supp} f$ such that $R_{\rho} f(\xi, \eta)=0$ for $|\xi|<1, \eta<1$.

It is obvious that local injectivity for real analytic and positive $\rho$ implies the following support theorem, $[\mathbf{B Q}]$. Assume $f$ has compact support in $\mathbf{R}^{2}$, let $K$ be a convex, compact set, and assume $R_{\rho} f(L)=0$ for all lines $L$ not intersecting $K$. Then $f=0$ in the complement of $K$.

On the other hand, the argument in the first part of the proof of Corollary 1.2 proves the following.

COROLlary 1.4. The set of smooth $\rho$ for which a support theorem does not hold for $R_{\rho}$ is dense in the set of smooth, positive weight functions.

\section{An example of local non-injectivity}

As in [Bo1] we shall prove Theorem 1.1 by first constructing the function $f$ and then constructing $\rho$ such that $R_{\rho} f=0$. It is clear that $f$ must be highly oscillatory, since $f$ must change sign on every line intersecting the interior of the support of $f$. To define $f$ we first choose $\varphi$ and $\psi$ in $C^{\infty}(\mathbf{R})$ such that

$$
\begin{aligned}
& \operatorname{supp} \varphi \subset[-a, a], \quad \varphi=1 \text { on }\left[-a_{0}, a_{0}\right] \\
& \operatorname{supp} \psi \subset[1-b, 1+b], \quad \psi=1 \text { on }\left[1-b_{0}, 1+b_{0}\right],
\end{aligned}
$$

where $a_{0}<a$ and $b_{0}<b$ will be chosen later. Set

$$
f_{k}(x, y)=\varphi\left(2^{k} x\right) \psi\left(2^{k} y\right) \cos 4^{k} x
$$

and choose $f$ as the lacunary Fourier series in $x$

$$
f=\sum_{k=0}^{\infty} f_{k} / k !
$$

It is clear that $f \in C^{\infty}\left(\mathbf{R}^{2}\right)$. 
LEMma 2.1. One can choose $a_{0}, a, b_{0}$, and $b$ such that $0<a_{0}<a, 0<b_{0}<b$, and

(a) $\quad \operatorname{supp} f \subset\{(x, y) ;|x| \leq y\}$;

(b) if the line $L(\xi, \eta)$ meets the support of $f_{k}$ and $|\xi| \leq 1$,

then $2 / 5<2^{k} \eta<8 / 5$, which can happen for at most two $k$;

(c) if $2 / 3 \leq 2^{k} \eta \leq 4 / 3$, then $\varphi\left(2^{k} x\right) \psi\left(2^{k}(\xi x+\eta)\right)=1$ for $|x| \leq a_{0} 2^{-k}$.

Proof. If $\varphi\left(2^{k} x\right) \psi\left(2^{k}(\xi x+\eta)\right) \neq 0$, then $\left|2^{k} x\right| \leq a$ and $2^{k}(\xi x+\eta) \in[1-b, 1+b]$, and if $|\xi| \leq 1$ this implies

$$
2^{k} \eta \in[1-b, 1+b]+[-a, a] \subset[1-b-a, 1+b+a] .
$$

If $4(1-b-a)>1+b+a)$, i. e. $a+b<3 / 5$, this can hold for at most two values of $k$. This shows that (b) holds if $a+b<3 / 5$. On the other hand, if $|\xi| \leq 1$, $\left|2^{k} x\right| \leq a_{0}$, and

$$
2^{k} \eta \in\left[1-b_{0}+a_{0}, 1+b_{0}-a_{0}\right],
$$

then $2^{k}(\xi x+\eta) \in\left[1-b_{0}, 1+b_{0}\right]$, and hence $\varphi\left(2^{k} x\right) \psi\left(2^{k}(\xi x+\eta)\right)=1$. Thus it is sufficient to choose $b_{0}-a_{0} \geq 1 / 3$ to get (c). For instance we can choose $b=1 / 2$, $b_{0}=2 / 5, a=1 / 16$, and $a_{0}=1 / 20$. Then $(x, y) \in \operatorname{supp} f_{k}$ implies $2^{k}|x| \leq a$ and $2^{k} y \geq 1-b$, hence $y /|x| \geq(1-b) / a=8$, so (a) certainly holds.

We shall choose $\rho(x, L)=1-c(L) f(x, y)$ for $(x, y) \in L$, that is,

$$
\rho(x, L(\xi, \eta))=\rho(x, \xi, \eta)=1-c(\xi, \eta) f(x, \xi x+\eta),
$$

where $c(\xi, \eta)=c(L(\xi, \eta))$ will be chosen so that

$$
\int_{L} f \rho d x=\int_{L} f d x-c(L) \int_{L} f^{2} d x=0
$$

for all $L$ with $|\xi|<1$ and $\eta<1$. This leads to

$$
c(L)=\int_{L} f d x / \int_{L} f^{2} d x, \quad \text { if }|\xi|<1 \text { and } 0<\eta<1,
$$

and $c(L)=0$ for all other lines $L$. The expression for $c(L)$ makes sense, since Lemma 2.1 (c) shows that $\int_{L} f^{2} d x>0$ when $0<\eta<1$. It is clear that $\rho(x, \xi, \eta)$ is smooth for $\eta>0$. To prove that $\rho(x, \xi, \eta)$ is also smooth near $\eta=0$ we shall prove that all derivatives of $\rho(x, \xi, \eta)$ tend to zero as $\eta$ tends to zero.

LEMMA 2.2. For any natural numbers $m$ and $p$ there exists a constant $C_{m, p}$ that is independent of $(\xi, \eta)$ (and hence independent of $k$ ) such that for $2^{-k} \sim \eta$ and $[\xi \mid<1$

$$
\left|\partial_{\xi}^{r} \partial_{\eta}^{s} \int_{L(\xi, \eta)} f d x\right| \leq C_{m, p} 2^{-k p} / k !, \quad r+s \leq m .
$$

Proof. Start from the expression

$$
\int_{L(\xi, \eta)} f_{k} d x=\int_{\mathbf{R}} \varphi\left(2^{k} x\right) \psi\left(2^{k}(\xi x+\eta)\right) \cos 4^{k} x d x
$$

and make $q$ partial integrations, which gives, if for instance $q$ is even,

$$
\int_{L(\xi, \eta)} f_{k} d x=(-1)^{q / 2} 4^{-k q} \int_{\mathbf{R}} \cos 4^{k} x \partial_{x}^{q}\left(\varphi\left(2^{k} x\right) \psi\left(2^{k}(\xi x+\eta)\right)\right) d x .
$$


If $q$ is odd, we get a similar expression with sin instead of cos. The absolute value of the integral can be estimated by $C_{q} 2^{k q}$, which gives

$$
\left|\int_{L(\xi, \eta)} f_{k} d x\right| \leq C_{q} 2^{-k q}
$$

Applying the derivative $\partial_{\xi}^{r} \partial_{\eta}^{s}$ adds a factor $\left(2^{k}|\xi|\right)^{r} 2^{s k} \leq 2^{k(r+s)}$, which gives

$$
\left|\partial_{\xi}^{r} \partial_{\eta}^{s} \int_{L(\xi, \eta)} f_{k} d x\right| \leq C_{m, q}^{\prime} 2^{k(m-q)} \leq C_{m, m+p}^{\prime} 2^{-k p}, \quad r+s \leq m,
$$

if we choose $q=m+p$. To finish the proof of the lemma we use Lemma 2.1 (b), which shows that

$$
\int_{L(\xi, \eta)} f d x=\int_{L(\xi, \eta)}\left(\frac{f_{k}}{k !}+\frac{f_{j}}{j !}\right) d x
$$

with $j=k \pm 1$ for $2^{-k} \sim \eta$, if $|\xi| \leq 1$.

LEMMA 2.3. We have the estimate

$$
\int_{L(\xi, \eta)} f^{2} d x \geq a_{0} 2^{-k-3} /(k !)^{2},
$$

if $2^{k} \eta \in[2 / 3,4 / 3]$.

Proof. Let $L(\xi, \eta)$ be given with $|\xi|<1$ and $0<\eta<1$. Choosing $k$ as in Lemma 2.1 (c) we have $f_{k}=\cos 4^{k} x$ on $L$ for $|x| \leq a_{0} 2^{-k}$ and by (b) we know that $f=f_{k} / k !+f_{j} / j$ ! on $L$, where $j=k \pm 1$. If $f_{k}$ and $f_{j}$ have the same sign, then $f^{2} \geq f_{k}^{2} /(k !)^{2}$. Since this must occur on $I=\left[-a_{0} 2^{-k}, a_{0} 2^{-k}\right]$ in a number of quarterperiods of $\cos 4^{k} x$ with total length at least slightly less than half of the length of $I$, we can certainly say that

$$
\int_{L(\xi, \eta)} f^{2} d x \geq \frac{1}{(k !)^{2}} \frac{1}{4} \int_{I} \cos ^{2} 4^{k} x d x \geq \frac{1}{(k !)^{2}} \frac{1}{4} \cdot \frac{1}{4}|I|=\frac{a_{0}}{(k !)^{2}} 2^{-k-3} .
$$

Lemma 2.4. If $2^{k} \eta \in[2 / 3,4 / 3]$ and $|\xi|<1$, then

$$
\left|\partial_{\xi}^{r} \partial_{\eta}^{s} \int_{L(\xi, \eta)} f^{2} d x\right| \leq \frac{C_{p}}{(k !)^{2}} 2^{k p}, \quad r+s \leq p,
$$

where $C_{p}$ depends only on $p$.

Proof. Differentiating the expression

$$
\int_{L(\xi, \eta)} f_{k}^{2} d x=\int_{\mathbf{R}} \varphi\left(2^{k} x\right)^{2} \psi\left(2^{k}(\xi x+\eta)\right)^{2} \cos ^{2} 4^{k} x d x
$$

we immediately obtain the estimate

$$
\left|\partial_{\xi}^{r} \partial_{\eta}^{s} \int_{L(\xi, \eta)} f_{k}^{2} d x\right| \leq C_{p} 2^{k p}, \quad r+s \leq p .
$$

By Lemma 2.1 (c) we know that

$$
\int_{L(\xi, \eta)} f^{2} d x=\int_{\mathbf{R}}\left(\frac{f_{k}}{k !}+\frac{f_{j}}{j !}\right)^{2} d x
$$

with $j=k \pm 1$, if $k$ is chosen so that $2^{k} \eta \in[2 / 3,4 / 3]$. Applying (2.6) and a similar estimate for derivatives of $f_{k} f_{k \pm 1}$ we obtain (2.5). 
Lemma 2.5. If $2^{k} \eta \in[2 / 3,4 / 3]$ and $|\xi|<1$, then

$$
\left|\partial_{\xi}^{r} \partial_{\eta}^{s} \frac{1}{\int_{L(\xi, \eta)} f^{2} d x}\right| \leq C_{p}(k !)^{2} 2^{k p}, \quad r+s \leq p,
$$

where $C_{p}$ depends only on $p$.

For the proof of Lemma 2.5 we shall use the following elementary fact.

LEMMA 2.6. Let $h$ be a smooth function on an open set $U \subset \mathbf{R}^{n}$ such that $h \geq 1$ on $U$ and

$$
\sup _{x \in U,|\alpha| \leq p}\left|\partial^{\alpha} h(x)\right| \leq M_{p}, \quad p=0,1, \ldots
$$

Let $N_{p}$ be a logarithmically convex sequence such that $N_{p} \geq M_{p}$ and $N_{0} \geq 1$. Then there exist constants $A_{p}$ that depend only on $p$ and on the dimension $n$ such that

$$
\sup _{x \in U,|\alpha| \leq p}\left|\partial^{\alpha} \frac{1}{h(x)}\right| \leq A_{p} N_{0}^{p} N_{p}, \quad p=0,1, \ldots
$$

Proof. An arbitrary partial derivative $\partial^{\alpha}(1 / h)$ of order $|\alpha|=p$ is equal to a sum of terms of the form

$$
C_{\alpha} \frac{1}{h^{q+1}} \prod_{\nu=1}^{q} \partial^{\beta_{\nu}} h
$$

where $\sum \beta_{\nu}=\alpha$ ( $\alpha$ and $\beta_{\nu}$ are multi-index) and $q \leq|\alpha|$. By the assumption we have $\left|\partial^{\beta_{\nu}} h\right| \leq M_{\left|\beta_{\nu}\right|} \leq N_{\left|\beta_{\nu}\right|}$, and by logarithmic convexity $N_{s} \leq N_{0}^{(p-s) / p} N_{p}^{s / p} \leq$ $N_{0} N_{p}^{s / p}$, hence

$$
\prod_{\nu=1}^{q}\left|\partial^{\beta_{\nu}} h\right| \leq N_{0}^{p} N_{p}
$$

The sum of all the coefficients $C_{\alpha}$ depends only on $p$ and the dimension $n$. This completes the proof.

Proof of Lemma 2.5. By Lemma 2.3 the function

$$
h(\xi, \eta)=a_{0}^{-1} 2^{k+3}(k !)^{2} \int_{L(\xi, \eta)} f^{2} d x
$$

satisfies $h(\xi, \eta) \geq 1$ if $2^{k} \eta \in[2 / 3,4 / 3]$. Let $B_{p}$ be a logarithmically convex sequence satisfying $B_{0} \geq 1$ and $B_{p} \geq 8 C_{p} / a_{0}$, where $C_{p}$ are the constants in Lemma 2.4. Applying Lemma 2.6 with $N_{p}=B_{p} 2^{k(p+1)}$ we obtain

$$
\left|\partial_{\xi}^{s} \partial_{\eta}^{t} \frac{1}{h(\xi, \eta)}\right| \leq A_{p} N_{0}^{p} N_{p} 2^{(p+1) k}, \quad s+t \leq p .
$$

Taking into account (2.8) we obtain (2.7).

END OF PROOF OF THEOREM 1.1. It remains only to show that the weight function $\rho(x, \xi, \eta)$ defined by $(2.1)$ is smooth on $\left\{(x, \xi, \eta) \in \mathbf{R}^{3} ;|\xi|<1, \eta<1\right\}$. We first prove that there are constants $C_{m, p}$ that depend only on $m$ and $p$ such that

$$
\left|\partial_{\xi}^{r} \partial_{\eta}^{s} c(\xi, \eta)\right| \leq C_{m, p} 2^{-k p} k !, \quad r+s \leq m,
$$

if $k$ is chosen so that $2^{k} \eta \in[2 / 3,4 / 3]$. Writing $c(\xi, \eta)$ as a product

$$
c(\xi, \eta)=\int_{L(\xi, \eta)} f d x \cdot \frac{1}{\int_{L(\xi, \eta)} f^{2} d x}
$$


we express an arbitrary derivative of order $m$ of $c(\xi, \eta)$ using Leibnitz' formula. In each term in the resulting sum we estimate a derivative of order $\leq m$ of $1 / \int_{L(\xi, \eta)} f^{2} d x$ by $C_{m}(k !)^{2} 2^{k m}$ using Lemma 2.5 . Replacing $p$ by $p+m$ in Lemma 2.2 we can estimate an arbitrary derivative of order $\leq m$ of $\int_{L(\xi, \eta)} f d x$ by

$$
C_{m, p} 2^{-k(m+p)} / k ! \text {. }
$$

Since the sum of all the coefficients in Leibnitz' formula depends only on $m$, the estimate (2.9) follows.

To finish the proof of the theorem we use the fact that

$$
\begin{aligned}
1-\rho(x, \xi, \eta) & =c(\xi, \eta) f(x, \xi x+\eta) \\
& =c(\xi, \eta)\left(\frac{f_{k}(x, \xi x+\eta)}{k !}+\frac{f_{j}(x, \xi x+\eta)}{j !}\right)
\end{aligned}
$$

with $j=k \pm 1$ if $k$ is chosen as indicated. We have to prove that all derivatives of $\rho(x, \xi, \eta)$ tend to zero as $\eta$ tends to +0 . An arbitrary derivative of order $\leq m$ of $f_{k}(x, \xi x+\eta)$ can be estimated by $C_{m} 4^{k m}$. By (2.9) it follows that an arbitrary derivative of order $\leq m$ of $c(\xi, \eta) f_{k}(x, \xi x+\eta) / k$ ! can be estimated by

$$
C_{m, p} 2^{-k p} C_{m} 4^{k m}, \quad \text { for } \eta \sim 2^{-k} \text {. }
$$

If we choose $p=2 m+1$ this expression becomes $\leq C \eta$, which proves the claim. This completes the proof of Theorem 1.1.

\section{A dense set of non locally injective $\rho$}

The proof of Theorem 1.3 depends on the simple observations that we can make the coefficient $c(\xi, \eta)$ in $(2.1)$ arbitrarily small by making the functions $f_{k}$ sufficiently oscillatory, and that nothing is changed in the arguments above if we replace the constant function 1 by an arbitrary smooth function $\rho_{0}(x, \xi, \eta)$.

Define $f=\sum f_{k} / k$ ! as before, where $f_{k}$ now depends on a parameter $\lambda$, that will have a fixed (large) value $\geq 1$ :

$$
f_{k}(x, y, \lambda)=\varphi\left(2^{k} x\right) \psi\left(2^{k} y\right) \cos \left(4^{k} \lambda x\right),
$$

and choose

$$
\rho(x, \xi, \eta)=\rho_{0}(x, \xi, \eta)-c(\xi, \eta, \lambda) f(x, \xi x+\eta, \lambda)
$$

where

$$
c(\xi, \eta, \lambda)=\int_{L(\xi, \eta)} f \rho_{0} d x / \int_{L(\xi, \eta)} f^{2} d x
$$

for $0<\eta<1$ and $c=0$ for $\eta \leq 0$. Then it is clear that $\int_{L} f \rho d x=0$ for all relevant lines $L$, so we only need to prove that $\rho$ is smooth and that $\rho \in V$ if $\lambda$ is sufficiently large.

LEMMA 3.1. Let $\rho_{0}(x, \xi, \eta)$ be an arbitrary smooth function on $\mathbf{R}^{3}$. For any natural numbers $m$ and $p$ there exists a constant $C_{m, p}$ that is independent of $(\xi, \eta)$ (and hence independent of $k$ ) and is independent of $\lambda \geq 1$ such that for $2^{-k} \sim \eta$ and $[\xi \mid<1$

$$
\left|\partial_{\xi}^{r} \partial_{\eta}^{s} \int_{L(\xi, \eta)} f \rho_{0} d x\right| \leq C_{m, p} 2^{-k p} \lambda^{-p} / k !, \quad r+s \leq m
$$


Proof. The proof is completely parallel to the proof of Lemma 2.2, so we only need to point out how the parameter $\lambda$ comes up in the formulas. It will clearly be enough to prove

$$
\left|\partial_{\xi}^{r} \partial_{\eta}^{s} \int_{L(\xi, \eta)} f_{k} \rho_{0} d x\right| \leq C_{m, p} 2^{-k p} \lambda^{-p}, \quad r+s \leq m,
$$

instead of (2.3). Making $q$ partial integrations in the expression for $\int_{L(\xi, \eta)} f_{k} \rho_{0} d x$ we obtain if $q$ is even

$$
\begin{gathered}
(-1)^{q / 2} \int_{L(\xi, \eta)} f_{k} \rho_{0} d x \\
=\left(4^{k} \lambda\right)^{-q} \int_{\mathbf{R}} \cos 4^{k} x \partial_{x}^{q}\left(\varphi\left(2^{k} \lambda x\right) \psi\left(2^{k}(\xi x+\eta)\right) \rho_{0}(x, \xi, \eta)\right) d x .
\end{gathered}
$$

Applying the derivative $\partial_{\xi}^{r} \partial_{\eta}^{s}$ with $r+s \leq m$ to this expression we obtain

$$
\left|\partial_{\xi}^{r} \partial_{\eta}^{s} \int_{L(\xi, \eta)} f_{k} \rho_{0} d x\right| \leq\left(4^{k} \lambda\right)^{-q} C_{m+q} 2^{k(m+q)}=C_{m+q} 2^{k m} 2^{-k q} \lambda^{-q},
$$

and choosing $q=m+p$ completes the proof.

END OF PROOF OF TheOREM 1.3. We have to show that, for arbitrary given $N$ and $\delta>0$, we can choose $\lambda$ so that

$$
\sup _{|\alpha| \leq N,|\xi|<1, \eta<1}\left|\partial^{\alpha}\left(\rho-\rho_{0}\right)\right|<\delta,
$$

where we have written $\partial^{\alpha}=\partial_{\xi}^{r} \partial_{\eta}^{s} \partial_{x}^{t}, \alpha=(r, s, t)$. The estimates for the derivatives of $\rho(x, \xi, \eta)-\rho_{0}(x, \xi, \eta)=c(\xi, \eta, \lambda) f(x, \xi x+\eta, \lambda)$ will be exactly the same as in the proof of Theorem 1.1 apart from the presence of the factor $\lambda^{-p}$ in the right hand side of (3.2). Indeed, the estimates for $\int_{L(\xi, \eta)} f^{2} d x$ and its inverse will be unaffected by the parameter $\lambda$, so for $c(\xi, \eta, \lambda)$ we get by means of Lemma 3.1 and Lemma 2.5

$$
\left|\partial_{\xi}^{r} \partial_{\eta}^{s} c(\xi, \eta, \lambda)\right| \leq \frac{C_{m, p} 2^{-k p} \lambda^{-p}}{k !} 2^{k m}(k !)^{2} \leq C_{m, p^{\prime}}^{\prime} 2^{k(m-p)} \lambda^{-p} k !, \quad r+s \leq m
$$

instead of (2.9). For the derivatives of $f_{k}(x, \xi x+\eta, \lambda)$ we have

$$
\left|\partial_{\xi}^{r} \partial_{\eta}^{s} \partial_{x}^{t} f_{k}(x, \xi x+\eta, \lambda)\right| \leq C_{m} 2^{(r+s) k}\left(4^{k} \lambda\right)^{t} \leq C_{m} 2^{2 m k} \lambda^{m}, \quad r+s+t \leq m .
$$

Thus, taking into account (2.10) we obtain with a new constant $C_{m, p}$

$$
\left|\partial^{\alpha}\left(\rho(x, \xi, \eta)-\rho_{0}(x, \xi, \eta)\right)\right| \leq C_{m, p} 2^{3 m k} 2^{-p k} \lambda^{-p}, \quad|\alpha| \leq m .
$$

With $m=N$ and $p=3 N$ this gives $\left|\partial^{\alpha}\left(\rho-\rho_{0}\right)\right| \leq C_{N, 3 N} \lambda^{-3 N}$, which we can make as small as we please by taking $\lambda$ sufficiently large. This completes the proof of Theorem 1.3.

\section{References}

[ABK] E. V. Arbuzov, A. L. Bukhgeim, and S. G. Kazantsev, Two-dimensional tomography problems and the theory of A-analytic functions, Siberian Adv. Math. 8 (1998), 1-20.

[Ba1] G. Bal, On the attenuated Radon transform with full and partial measurements, Inverse Problems 20 (2004), 399-418.

[Ba2] G. Bal and A. Tamasan, Inverse source problems in transport equations, SIAM J. Math. Anal. 39 (2007), 1963-1971.

[Bo1] J. Boman, An example of non-uniqueness for a generalized Radon transform, J. Anal. Math. 61 (1993), 395-401. 
[Bo2] J. Boman, A local uniqueness theorem for weighted Radon transforms, Inverse Probl. Imaging 4 (2010), 631-637.

[BQ] J. Boman and E. T. Quinto, Support theorems for real-analytic Radon transforms, Duke Math. J. 55 (1987), 943-948.

[BS] J. Boman and J.-O. Strömberg, Novikov's inversion formula for the attenuated Radon transform - a new approach, J. Geom. Anal. 14 (2004), 185-198.

[G] S. Gindikin, A remark on the weighted Radon transform on the plane, Inverse Probl. Imaging 4 (2010), 649-653.

[Fi] D. Finch, The attenuated X-ray transform: recent developments, in G. Uhlmann (ed.): "Inside out: Inverse problems and Applications", Cambridge Univ. Press 2003.

[Fo] A. S. Fokas, Generalized Fourier transforms, inverse problems, integrability in $4+2$, in "Mathematical methods for imaging and inverse problems", 55-64, ESAIM Proc. 26, EDP Sci., Les Ulis 2009.

[KB] S. G. Kazantsev and A. A. Bukhgeim, Inversion of the scalar and vector attenuated X-ray transforms in a unit disc, J. Inverse Ill-posed Probl. 15 (2007), 735-765.

[Na] F. Natterer, Inversion of the attenuated Radon transform, Inverse Problems 17 (2001), $113-119$.

[No] Novikov, R. G., An inversion formula for the attenuated X-ray transform, Ark. Mat. 40 (2002), 145-167.

[S] R. S. Strichartz, Radon inversion - variations on a theme, Amer. Math. Monthly, 89 (1982), 377-384, 420-423.

Department of Mathematics, Stockholm University, SE-10691 Stockholm, Sweden

E-mail address: jabo@math.su.se 